\title{
Implementing the Patient-Centered Medical Home: Observation and Description of the National Demonstration Project
}

\author{
Elizabeth E. Stewart, PbD \\ Paul A. Nutting, MD, MSPH \\ Benjamin F. Crabtree, PbD \\ Kurt C. Stange, $M D, P b D$ \\ William L. Miller, MD, MA \\ Carlos Roberto Jaén, MD, PbD
}

\begin{abstract}
PURPOSE We provide an overall description of the National Demonstration Project (NDP) intervention to transform family practices into patient-centered medical homes.

METHODS An independent evaluation team used multiple data sources and methods to describe the design and implementation of the NDP. These included direct observation of the implementation team and project meetings, site visits to practices, depth interviews with practice members and implementation team members, access to practice communications (eg, telephone calls, e-mails), and public domain materials (eg, the NDP Web site).
\end{abstract}

RESULTS The American Academy of Family Physicians created a new division called TransforMED, which launched the 24-month NDP in June 2006. From 337 family medicine practices completing an extensive online application, 36 were selected and randomized to a facilitated group, which received tailored, intensive assistance and services from TransforMED, or a self-directed group, which received very limited assistance. Three facilitators from diverse backgrounds in finance, practice management, and organizational psychology used multiple practice change strategies including site visits, e-mails, metrics, and learning sessions. The self-directed practices worked primarily on their own, but selforganized a retreat midway through the project. The intervention model for the project evolved to be consistent with the emerging national consensus principles of the patient-centered medical home. The independent evaluation team studied the NDP and provided ongoing feedback to inform the implementation process.

CONCLUSIONS The NDP illustrates that complex practice change interventions must combine flexibility in the intervention model, implementation strategy, and the evaluation, in order to maximize ongoing learning.

Ann Fam Med 2010;8(Suppl 1):s21-s32. doi:10.1370/afm.1111.

Conflicts of interest: The authors' funding partially supports their time devoted to the evaluation, but they bave no financial stake in the outcome. The authors' agreement with the funders gives them complete independence in conducting the evaluation and allows them to publish the findings without prior review by the funders. The authors bave full access to and control of study data. The funders bad no role in writing or submitting the manuscript.

\section{CORRESPONDING AUTHOR}

Elizabeth E. Stewart, PhD

National Research Network

American Academy of Family Physicians 11400 Tomahawk Creek Pkwy

Leawood, KS 66211-2680

estewart@aafp.org

\section{INTRODUCTION}

$\mathrm{T}$ The Future of Family Medicine (FFM) project was a collaborative initiative of 7 national family medicine organizations "to develop a strategy to transform and renew the specialty of family medicine to meet the needs of patients in a changing health care environment."1 A series of task force reports theorized that a "New Model" of practice would increase patient access and satisfaction, improve efficiency and work flow, produce higher-quality measures of care, maximize use of technology, and enhance working conditions for physicians and staff, all while improving the financial viability of the practice. ${ }^{2,3}$ The FFM report, published in 2004, proposed the creation of a national-level organization to support and guide family medicine practices in their efforts to transform to this new model of care. As a result, the American Academy of Family Physicians (AAFP) funded the 2-year National Demonstration Project 
(NDP) and created TransforMED in 2005 as an AAFP division to conduct the project.

The design and implementation of the NDP were the sole responsibility of TransforMED. Overall, the NDP was designed as a demonstration project of newmodel practice in which everything possible was done to implement the full model using facilitators and full access to necessary support and resources. A secondary consideration was to determine whether the intensity of the intervention influenced its effectiveness; thus, a clinical trial was developed in which practices were randomized into 2 levels of intervention. It should be noted that the intervention evolved as experience grew and was not constrained by a protocol in the usual style of a randomized controlled trial.

This article provides background for understanding the NDP and its context, and complements patient and practice outcomes reported elsewhere in this supplement. ${ }^{4-6}$ We report our methods of observation and describe the chronology of the project, the development and evolution of the guiding model, the facilitation strategies, and changes to the intervention as it evolved over time. ${ }^{7,8}$

\section{EVALUATION METHODS}

The TransforMED board of directors contracted with an independent evaluation team (the authors of this article), the Center for Research in Family Medicine and Primary Care, to study the NDP model and the implementation process. An explicit purpose of the evaluation was to provide real-time, ongoing analysis and feedback in order to stimulate both the TransforMED leadership and facilitators to engage in self-evaluation, reflection, and learning, and thereby maximize the potential for the intervention to have an impact. This evaluation strategy focused on engaging stakeholders at TransforMED and its board of directors, the general public, and NDP practices, and as such has many resemblances to empowerment evaluation. ${ }^{9}$ The evaluation team had frequent conversations with the practice facilitators, especially in the first year, and both conference calls and occasional faceto-face meetings created a mutual iterative process of feedback, reflection, discussion, and brainstorming. Analysis reports were submitted quarterly to the TransforMED board and posted for public dissemination on the TransforMED Web site (http://www.transformed. com/evaluatorsReports/index.cfm). The interactive nature of the evaluation at times appeared to influence the project development (as described below) and thus should be considered to be part of the intervention.

As the evaluation team, we used a variety of data sources to construct a detailed description of the proj- ect design and implementation. This data collection took place in parallel with TransforMED's intervention efforts to assist practices in implementing the NDP model (Figure 1). One member of the evaluation team (E.E.S.) was embedded within the TransforMED office, and her firsthand direct observations supplemented additional information available through public domains (Web sites, media, etc) and communications provided by the TransforMED team. We also observed the interface between TransforMED and the participating practices. We used multiple data elements for this process: site visits and depth interviews with every participating practice (conducted by E.E.S.); direct observations at learning sessions and conference calls; periodic interviews with facilitators; compilation and synthesis of extensive e-mail streams between and among practices and facilitators; and documentation of the NDP model components implemented by the practices, gathered from both facilitator and practice interviews. Finally, we used opportunities to interact with TransforMED leadership, facilitators, and NDP practices at learning sessions for "member-checking" to determine how our observations correlated with those of TransforMED and practice participants. Nonetheless, we acknowledge that the following represents an external observation and our interpretation of the nuances of the NDP.

\section{RESULTS}

Over the course of the NDP, we made observations about the formation and growth of TransforMED's approach to launching the project, including selection of practices, preparation of the facilitators, changes in the NDP conceptual model, and progression of the implementation strategy. We also observed activities in the self-directed practices. We describe components of the NDP intervention in both facilitated and selfdirected practices that go well beyond the mere presence or absence of a facilitator.

TransforMED was established as a division of the AAFP with full responsibility for the design and conduct of the NDP. Oversight was provided by a board of directors. TransforMED staff included a chief executive officer, an executive director, 3 practice enhancement facilitators, and 2 support staff (http:// transformed.com/ndp.cfm). The 3 facilitators were intentionally recruited from distinctly different backgrounds (finance/management, practice operations, and organizational psychology), bringing different skill sets and strengths to the project. Three months of training included an introduction to theories of practice change (eg, practices as complex adaptive systems), as well as the more technical instruction about the model components (eg, discussions with national experts on 
health information technology [HIT] and change management). The facilitator training continued throughout the project with ongoing discussions and visits or conference calls with experts and outside consultants. Facilitators also spent several days of intense training with the evaluation team, focusing on qualitative data collection for the baseline site visits (eg, field notes, interviews, observation checklists).

\section{Practice Participants}

A TransforMED Technical Advisory Committee evaluated 337 complete online applications and selected a sample of 36 practices that had high potential to be successful in implementing the NDP model and as a group were varied in geography, size, practice age, physician and staff structure, ownership arrangement, and scope of practice. Details of the recruitment process are published elsewhere. ${ }^{8}$ Once the practices were selected, the evaluation team randomized them to facilitated and self-directed groups.

It was apparent to us that the participating practices were exceptional in several important respects. For example, all practices had at least 1 highly motivated physician, $70 \%$ had an electronic medical record (EMR) at baseline, all were familiar with the FFM report, and most had many NDP model components already in place. A further description of practices' baseline characteristics appears elsewhere in this supplement. ${ }^{6}$ Although all 36 initially agreed to participate, 4 practices ( 3 self-directed, 1 facilitated) withdrew during the first 9 months of the 2 -year project; thus, data on these practices are limited. An additional practice (facilitated) withdrew near the end. A description of the facilitated and self-directed practices can be found in Table 1, while Table 2 gives characteristics of the practices that withdrew.

\section{Figure 1. Flow of events in the NDP.}

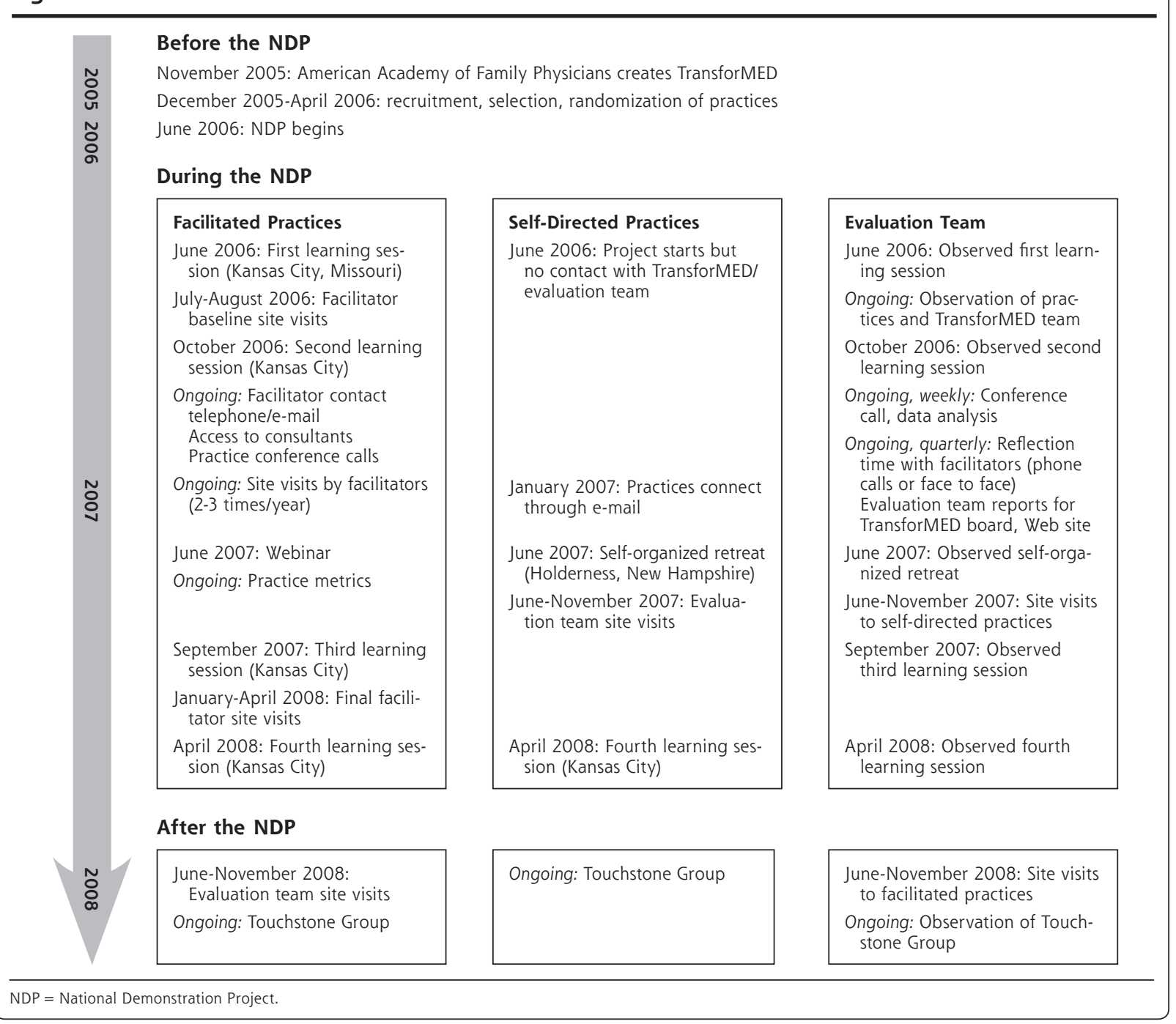




\begin{tabular}{|c|c|c|}
\hline Characteristic & $\begin{array}{c}\text { Facilitated }^{a} \\
\%\end{array}$ & $\begin{array}{c}\text { Self-Directed } \\
\%\end{array}$ \\
\hline \multicolumn{3}{|l|}{ Age of practice, $y$} \\
\hline$\leq 5$ & 35 & 33 \\
\hline $6-10$ & 24 & 5 \\
\hline $11-20$ & 6 & 29 \\
\hline$>20$ & 35 & 33 \\
\hline \multicolumn{3}{|l|}{ Size } \\
\hline Solo ( \pm midlevel clinicians) & 35 & 22 \\
\hline Small (2-3 physicians) & 24 & 12 \\
\hline Medium (4-6 physicians) & 17 & 44 \\
\hline Large ( $\geq 7$ physicians) & 24 & 22 \\
\hline \multicolumn{3}{|l|}{ Setting } \\
\hline Rural & 29 & 33 \\
\hline Suburban & 53 & 55 \\
\hline Urban & 18 & 11 \\
\hline \multicolumn{3}{|l|}{ Scope of practice } \\
\hline Home visits & 29 & 50 \\
\hline Prenatal care & 23 & 55 \\
\hline Hospital care & 59 & 66 \\
\hline Labor and delivery & 23 & 44 \\
\hline \multicolumn{3}{|l|}{ Ownership structure } \\
\hline Physician owned & 59 & 61 \\
\hline $\begin{array}{l}\text { Health or hospital system } \\
\text { owned }\end{array}$ & 41 & 39 \\
\hline \multicolumn{3}{|l|}{$\begin{array}{l}\text { Health information } \\
\text { technology }\end{array}$} \\
\hline Electronic medical record & 76 & 61 \\
\hline Electronic prescribing & 70 & 44 \\
\hline Practice Web site & 35 & 50 \\
\hline Interactive patient portal & 5 & 5 \\
\hline \multicolumn{3}{|l|}{ Access to care } \\
\hline Same-day appointments & 53 & 61 \\
\hline Group visits & 6 & 11 \\
\hline e-Visits & 17 & 5 \\
\hline \multicolumn{3}{|l|}{ Population management } \\
\hline Disease registry & 0 & 5 \\
\hline Team model & 12 & 11 \\
\hline
\end{tabular}

\section{The NDP Model of Care}

TransforMED leadership designed a model that was intended to guide the implementation process for facilitators and practices alike (Figure 2). This original model was patterned on the FFM report. ${ }^{1,3}$ Over the course of the NDP, changes were made to model details. Publication of the joint statement on the patient-centered medical home $(\mathrm{PCMH})^{10}$ relatively early in the NDP led to refinements and reorganization of the model framework. Feedback from The Commonwealth Fund emphasized patient-centered care, which was incorporated more explicitly in the model. ${ }^{11-13}$ Ongoing refinements to the model over the 2-year project more clearly specified the different components relating to clinical and practice operations, whereas the core of the model continued to focus on the continuous relationship with patients.

Even after multiple revisions, however, the revised NDP model (Figure 3) still appeared to emphasize technology at the expense of the pillars of primary care (easy access to first-contact care, comprehensive care, coordination of care, and personal relationship over time). The original model was based on FFM recommendations, which included collection of patient data ${ }^{1}$, however, the NDP model did not incorporate changes based on patient feedback, nor did patient input play a role in the facilitator decisions and strategies or the model components that practices implemented. The model broadly highlighted team care but remained fairly physician-centric with limited recognition of the configuration of skills necessary for patient care not directed by a physician (eg, community-based programs, pharmacists, physical therapists). Finally, the model did not include mental health or specific components to coordinate the practice with the larger medical neighborhood.

\section{Evolution of the NDP Intervention}

In facilitated practices, the NDP intervention was purposefully intended to do everything reasonably possible to assist the practices in implementing as

Table 2. Baseline Characteristics of Practices That Dropped Out of the NDP

\begin{tabular}{|c|c|c|c|c|}
\hline $\begin{array}{l}\text { Practice and } \\
\text { Group }\end{array}$ & Dropout Date & Size, Location, Ownership & $\begin{array}{l}\text { Practice } \\
\text { Age, y }\end{array}$ & Reason for Dropout \\
\hline D-1: Self-directed & October 2006 & $\begin{array}{l}\text { Large, metropolitan, system } \\
\text { owned }\end{array}$ & 17 & $\begin{array}{l}\text { Restructuring within system closed the original fam- } \\
\text { ily medicine practice that applied to the NDP }\end{array}$ \\
\hline D-2: Self-directed & March 2007 & $\begin{array}{l}\text { Medium, suburban, physician } \\
\text { owned }\end{array}$ & 25 & $\begin{array}{l}\text { Practice said they had too many competing } \\
\text { demands to participate in NDP data collection }\end{array}$ \\
\hline D-3: Self-directed & April 2007 & Solo, rural, physician owned & 2 & $\begin{array}{l}\text { Local hospital closed, forcing close of practice; phy- } \\
\text { sician joined practice in another town }\end{array}$ \\
\hline D-4: Facilitated & March 2008 & Solo, suburban, physician owned & $<1$ & $\begin{array}{l}\text { Physician owner closed original practice and joined } \\
\text { another }\end{array}$ \\
\hline
\end{tabular}

$\mathrm{IRB}=$ institutional review board; NDP = National Demonstration Project.

Note: One facilitated practice dropped out of the NDP when the health system IRB did not approve inclusion in the study. All data related to this practice were expunged. 
Figure 2. The original "New Model" of practice as conceptualized by TransforMED.

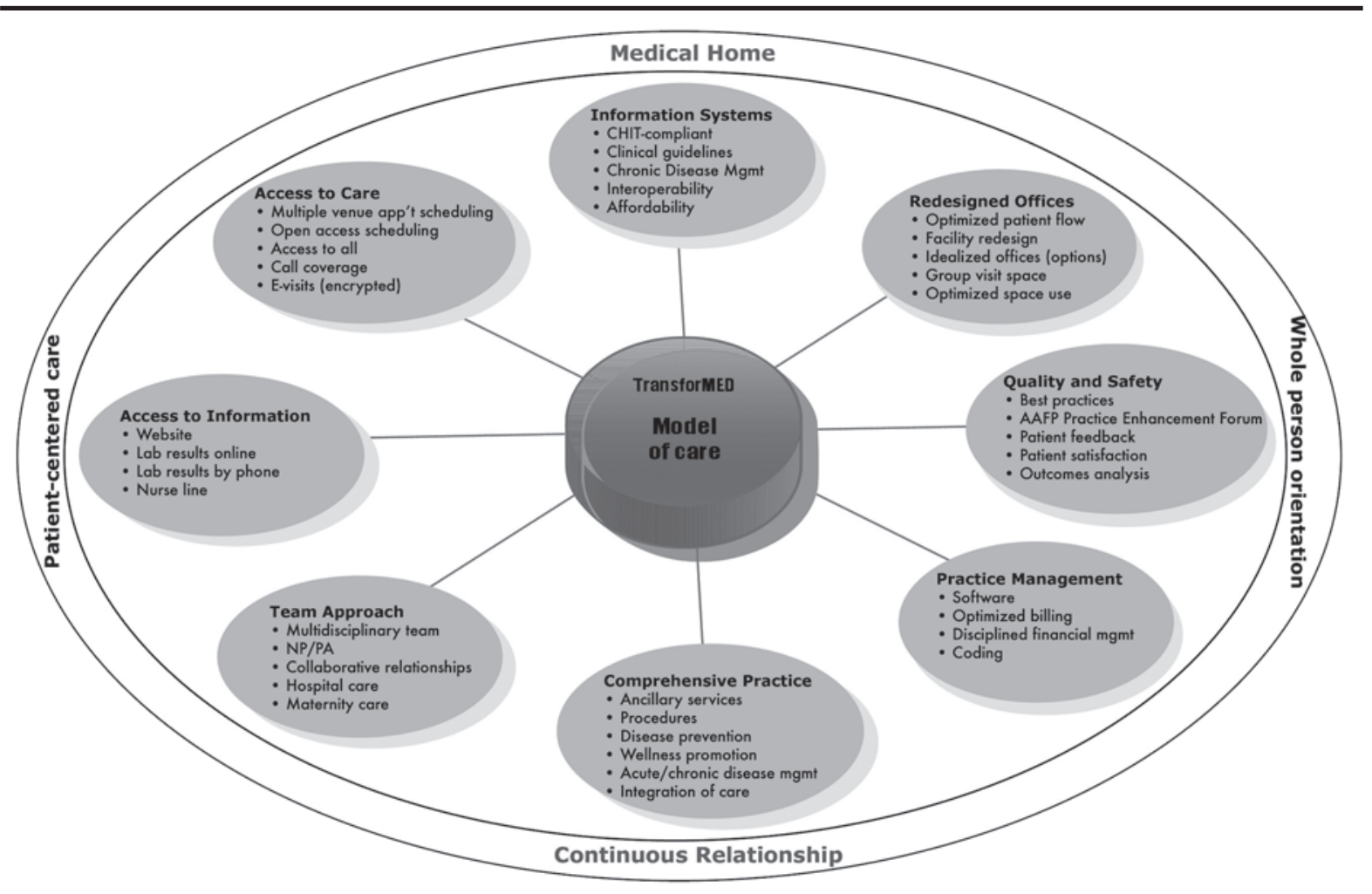

AAFP = American Academy of Family Physicians; $a p p ' t=$ appointment; $C H I T=$ Center for Health Information and Technology $($ AAFP $;$ mgmt $=$ management; $\mathrm{NP}=$ nurse practitioner; $\mathrm{PA}=$ physician assistant.

Reprinted with permission from TransforMED, Leawood, Kansas.

many model components as possible. Consequently, the NDP efforts were not constrained by a fixed protocol but evolved in real time based on the experiences of the facilitators and input of the evaluation team. During the first year, the facilitators tended to work exclusively with their panel of 6 practices. With the frenetic pace at the project's onset, there were few opportunities for sharing concerns and mutual assistance. The facilitators did have limited time (in telephone calls and some face-to-face meetings) for shared reflection with the evaluation team, however.

Although the evaluation team's interaction with facilitators was planned to be mainly for data collection, we began to observe some changes in how the facilitators interacted with each other and their practices when given protected time and space to pause, reflect, brainstorm, and share. For example, one facilitator felt "stuck" with a practice that continued to backslide while others moved forward. After conversations with the evaluation team and other facilitators, this facilitator decided to try an all-practice retreat to repair the practice's relationships and revitalize its core motivation. The retreat results were promising, word spread among other practices, and the facilitators began to work together on staging retreats for other practices that requested the service. These efforts led to sharing of skills in other areas, and during the second year, facilitators made "guest" site visits to others' practices in order to offer more specialized guidance, such as financial management and practice efficiency. In this way, we as the evaluation team observed the intervention evolve from solo efforts by facilitators at baseline to eventually more shared endeavors congregating all skills and strategies. We also realize that our real-time evaluation activities played a role in this evolution.

\section{NDP Intervention for Facilitated Practices}

\section{Chronology}

Kick-off Learning Session (June 2006). TransforMED formally launched the NDP in June 2006 with a kick-off learning session in Kansas City, Missouri, for the 18 facilitated practices. The project paid for the attendance of 2 practice representatives-typically, a lead physician and a practice manager - at this and all following sessions. The agenda tilted toward a "shock and awe" approach with speeches by AAFP and Trans- 
Figure 3. The revised model of practice tested in the NDP, as formalized in 2008.

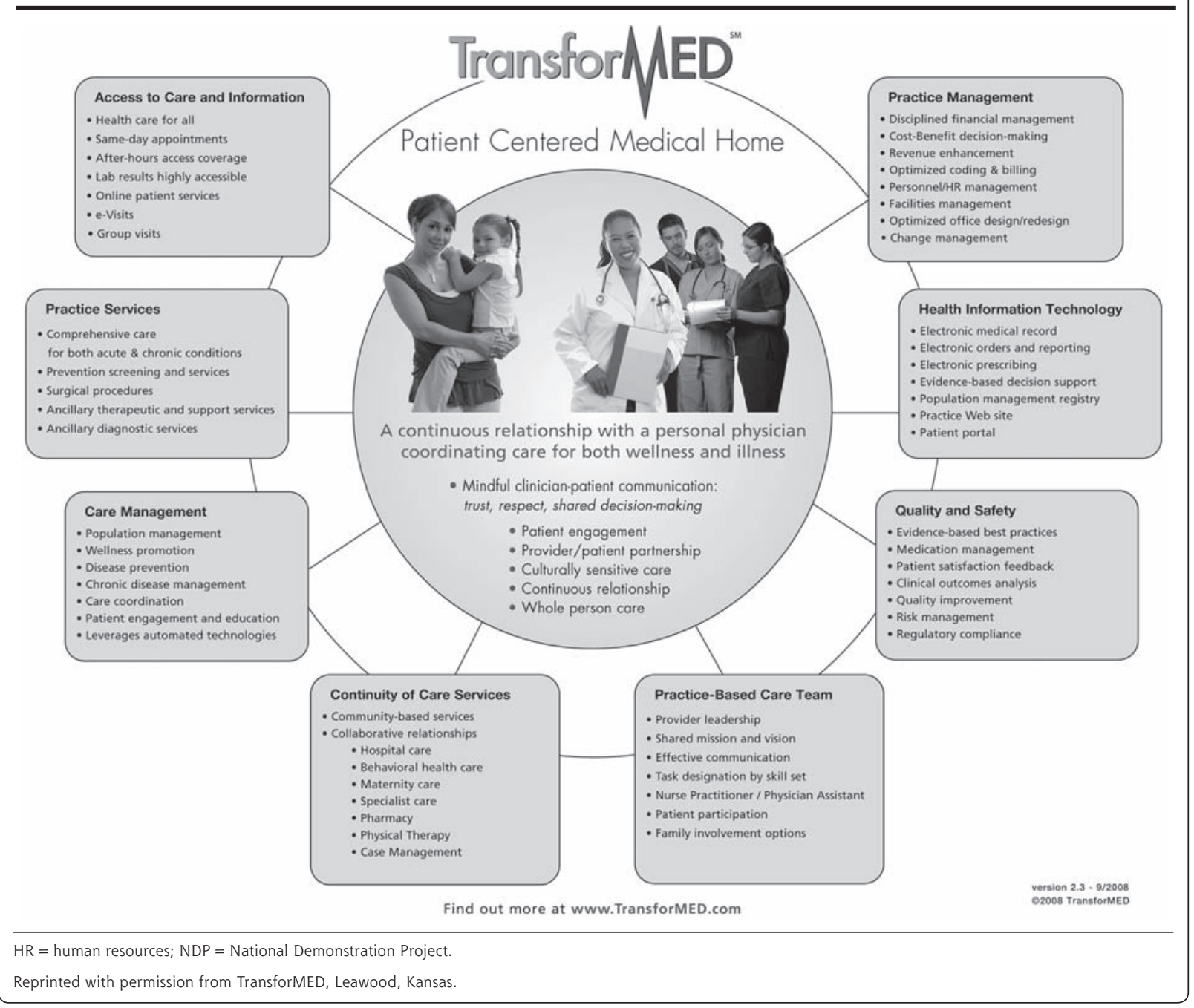

forMED leadership, presentations by national-level consultants, and demonstrations of different technologies. Although the style was heavily didactic, participants had some opportunities to share introductions and ideas with their facilitator and other practices. We observed a mood that was upbeat and confident, energetic and enthusiastic, with many participants stating they had already put many pieces of the model in place or would do so as soon as they returned home.

Initial Site Visit and Baseline Assessment (Summer 2006). Immediately after the kick-off session, the facilitators visited each practice for an initial site visit and baseline assessment. These visits lasted from 2 to 4 days, depending on the size of the practice and the work style of the facilitator. By this time, many practice leaders who attended the kick-off learned on their return that others in their practice did not share their excitement, and buy-in was not a given. The facilitators therefore often found themselves needing to regener- ate the excitement that had waned since the kick-off, giving practice leaders a reality check in regard to what could really be done, or both.

The facilitators collected baseline data that served the dual purpose of (1) developing a practice assessment to guide future facilitation work and (2) providing the evaluation team with an initial look at the practice from the facilitator's perspective. The visits also gave the facilitators an opportunity to form relationships with key members of the practice and develop a shared understanding of practice preference for model components and desired time lines. Although most facilitator time during the first visit was devoted to data collection, the facilitators also taught effective meeting techniques with a specific emphasis on daily, brief meetings known as huddles. ${ }^{14}$ Many practices stated they had tried meetings in the past and "nothing ever got done," so often the facilitator would model the desired meeting style and attend for several 
weeks by telephone to reinforce guidelines. The ability of a practice to hold effective, regular meetings was seen as an early win by practices and facilitators alike.

Interim Learning Sessions (October 2006 and September 2007). Within the first 6 months, the facilitators again noted a deterioration of energy and enthusiasm in practice leaders as they faced the daily reality of seeing patients, keeping their practice afloat, and addressing the many changes outlined by the intervention. The facilitators also noticed gaps between the consultants' high-level presentations at the kick-off session and the challenges of actual implementation at the ground floor. Finally, they sensed that this type of change was harder than anything the practices had attempted before, even among the few practices with previous continuous quality improvement (CQI) experience. They therefore planned the second learning session just 5 months after the first as an opportunity to reenergize the practices and revisit model components in more concrete ways.

It was during this second learning session that we observed an early evolution of practice learning. Although the agenda at this second learning session still included national-level consultants, an explosion of crosstalk among the practices permanently shifted the NDP learning environment from didactic to interactive, prompting one practice leader to note, "We are the experts in practice change ... we have to learn from each other."

The atmosphere of collaborative teaching continued with the third learning session a year later, and by this time, several practices were willing to pay the extra expense to bring additional practice members. Some practice leaders appeared to recognize the future value of exposing others in the practice to the infectious excitement and positive energy of the learning sessions, perhaps as a way to increase the needed "change agents" back home at the practice.

Final 6 Months to Project's End (May 2008). During the last 6 months of the NDP, facilitated practices prepared for life without their facilitator. Deep bonds were apparent, and some practice members needed reassurance that their facilitator would still be available for consultation or even just conversation once the project ended. Another evolutionary step occurred as practices began to think beyond the NDP and explore other challenges and opportunities. Some began to actively pursue the medical home recognition process offered by the National Committee for Quality Assurance (NCQA); others found additional CQI or medical home projects sponsored by their state, a grant, or insurance companies. Many physician leaders publicly took the step from project participant to change advocate and role model, speaking at conferences and meetings on behalf of transformative change.

\section{Change Strategies Used by Facilitators}

We observed that facilitators used multiple strategies to keep the practices engaged and focused (Table 3 ) and practices reported multiple factors that motivated them (Table 4). A few strategies are described in further detail below.

\section{Table 3. Strategies Used to Keep Practices Engaged}

\begin{tabular}{|c|c|}
\hline Facilitated Practices & Self-Directed Practices \\
\hline Access to NDP Web site, e-mail listserv & $\begin{array}{l}\text { Access to NDP Web site, } \\
\text { e-mail listserv }\end{array}$ \\
\hline 4 NDP on-site learning sessions & 1 self-organized retreat \\
\hline Daily access to facilitator by telephone/e-mail & $\begin{array}{l}\text { Evaluation team presence } \\
\text { at retreat }\end{array}$ \\
\hline Facilitator site visits (3-6 total) & 1 NDP on-site learning session \\
\hline $\begin{array}{l}\text { Evaluation team site visit ( } 1 \text { total) } \\
\text { Limited access to consultants (national experts) }\end{array}$ & $\begin{array}{l}\text { Evaluation team site visit } \\
\quad(1 \text { total) }\end{array}$ \\
\hline Discounted HIT and facilitator assistance with implementation & \\
\hline $\begin{array}{l}\text { Assistance with practice management (eg, HR, meetings, poli- } \\
\text { cies and protocols) }\end{array}$ & \\
\hline $\begin{array}{l}\text { Assistance with financial management (eg, budgets, forecast- } \\
\text { ing, collections) }\end{array}$ & \\
\hline $\begin{array}{l}\text { Assistance with change management (eg, conflict resolution, } \\
\text { all-staff retreats, communication, teamwork) }\end{array}$ & \\
\hline $\begin{array}{l}\text { Assistance with personal development (eg, leadership coaching, } \\
\text { communication) }\end{array}$ & \\
\hline $\begin{array}{l}\text { Assistance with practice metrics (eg, collection and analysis } \\
\text { of own data for improvement) }\end{array}$ & \\
\hline Evaluation team connection to facilitators & \\
\hline
\end{tabular}

$\mathrm{HIT}=$ health information technology; $\mathrm{HR}=$ human resources; NDP = National Demonstration Project.

\begin{tabular}{|ll}
\hline Table 4. Motivating Factors as & Reported by Practices \\
\hline Facilitated Practices & Self-Directed Practices \\
\hline $\begin{array}{l}\text { Prestige of being part of important proj- } \\
\text { ect; being in national spotlight }\end{array}$ & $\begin{array}{c}\text { Prestige of being part of important project; being } \\
\text { in national spotlight }\end{array}$ \\
$\begin{array}{c}\text { Support of other practices and } \\
\text { TransforMED }\end{array}$ & $\begin{array}{c}\text { Support of other practices, especially after midway } \\
\text { retreat }\end{array}$ \\
Accountability to facilitator; external & Desire to perform well despite self-directed status \\
support and assistance & Evaluation team site visit \\
\hline NDP = National Demonstration Project. & \\
\hline
\end{tabular}


Site Visits. Depending on the needs of the practice and the work style of the facilitator, the facilitators visited their assigned practices about twice a year after the initial site visit. Such visits typically lasted 1 to 3 days and often included formal and informal meetings with members at all levels of the practice; observation of work-flow processes with suggestions for improvement; a review of practice finances; assistance, meetings with vendors or outside stakeholders, or both; and problem solving and brainstorming on issues ranging from the most user-friendly disease registry to the best way to deal with a chronically tardy employee.

Constant Communication. Between site visits, the facilitators were assertive in the initiation and maintenance of communication by telephone and e-mail. Whereas some practices preferred more autonomy, others had key members who contacted the facilitator multiple times a day. Asynchronous e-mail was by far the preferred form of communication, especially with physicians. The facilitators often served as "connectors" and used e-mail to formally introduce practice members to other NDP practice members when it appeared both could benefit from direct communication with each other.

Listserv. At the start of the NDP, TransforMED created 2 private, password-accessible message boards for separate use by the facilitated and self-directed groups. Within months, the physicians said the message board required too much navigation time and asked for a simple e-mail listserv instead. Communication gained momentum as the NDP progressed, with some threads lasting days or even weeks. Topics ranged from concrete questions about preauthorizations or an EMR function to the more nuanced, personal statements on patient-centered care or practice cohesion.

Conference Calls. The 3 panels of facilitated practices participated in monthly, hour-long conference calls within their own group. The calls allowed the participants (lead physician and often practice manager) to give progress reports, share challenges and successes, ask for or give advice, and remain accountable to the facilitator and group at large. The facilitators constantly encouraged participation from all levels of the practice. The second year, the facilitators still attended, but the participants took turns setting the agenda and moderating the calls.

HIT Assistance. TransforMED did not provide any discounts on EMR products; however, facilitated practices had available to them other discounted HIT products and services, including Web sites, e-visits, patient portals, and disease registries. The decision to implement HIT was based on practice interest and the ability to fulfill initial requirements (eg, if a practice wanted e-visits, it first needed an interactive Web site). Web sites were considered easy wins by many practices, as the process of creating a site lent itself to teamwork and wide staff participation. TransforMED used a company whose product allowed practices to either create their own Web site or integrate interactive patient functions into an existing Web site. The facilitators served as liaisons during the Web site development and also with vendors of other HIT products, such as stand-alone disease registries. As liaisons, the facilitators coordinated vendor-practice conference calls and Web-based demonstrations, in addition to arranging calls with other practices for peer advice.

Consultants. The facilitators had access to almost a dozen national-level consultants with expertise on topics such as group visits, HIT implementation and use, virtual communication, quality and safety, patient satisfaction and patient-centered care, and efficiency and work-flow redesign. Many of these consultants spoke at the kick-off learning session, contributing to levels of high excitement and expectations. TransforMED expressed initial financial concerns about the possible overuse of consultants, but both facilitators and practices rapidly realized that the consultants-knowledgeable at the broad scale or in niche markets-seemed to have limited utility at the very granular, specific level of the NDP practice. For example, a consultant could speak on the benefits of group visits and explain different models, but the practices needed help with the sheer logistics of setting up the room or reconciling medications in a group setting. Fairly quickly, the practices began to view each other as the best sources for advice and consultation.

NDP Web Site. The TransforMED NDP Web site served as both a toolkit of resources for the NDP practices and a place to highlight and showcase NDP practice efforts. Physicians and practice members agreed to be interviewed or submitted their own thoughts on varied topics such as group visits, leadership, teamwork, work-life balance, and the medical home concept. During the project, TransforMED held the philosophy that the Web site should offer "1-stop shopping" for any practice looking for tools on practice transformation, and thus, all contents (articles, working papers, sample documents, etc) were available to anyone.

Practice Metrics. During the first year, the NDP facilitators noted that many practices were not quantitatively assessing practice performance. Although several practices had experience in collecting and assessing clinical indicators, usually as part of a health system effort or external quality improvement project, very few were monitoring practice operations. TransforMED leadership determined that implementing a uniform set of metrics would be helpful to the practices and would provide TransforMED with outcomes for future 
business use. A metrics manager was therefore hired to assist practices with the design, collection, and analysis of metrics, including patient cycle time and patient satisfaction, staff satisfaction, prevention and screening measures, and same-day scheduling. The metric exercises were independent of the evaluation team's data collection efforts and analysis, but a TransforMED working paper on the metrics can be found online (http://www.transformed.com/workingpapers.cfm).

Practice Staff Retreats. As previously mentioned, the idea of using a practice retreat as a change strategy emerged from a shared discussion between the TransforMED facilitators and evaluation team. The facilitator with a background in organizational psychology developed a retreat outline that included an initial assessment of the practice and its history; intense prework with confidential interviews; facilitated group conversations to allow honest dialogue; and consistent follow-through to ensure the sustainability of changes sparked during the retreat. Some retreats had mixed results, as the facilitators worked to refine this strategy, but others appeared to be a real turning point for a practice, the first big step in moving from a dysfunctional, disparate group of individuals to a more cohesive team unified by the goal of practice change.

\section{NDP Intervention for the Self-Directed Practices} First Year

The 18 self-directed practices started the NDP with little more than the knowledge that they were part of a national project. They received the NDP Web site address, contact information for TransforMED staff, and instructions on how to use their private message board and later their listserv. There was no communication among the practices for the first 6 months, and their first real contact with the project was a visit by the evaluation team nurse performing medical record audits. The nurse reported that for many practices, her visit marked the first time they had seen the TransforMED model.

Later, during interviews with the evaluation team and at the final learning session, members of the selfdirected practices admitted their first response to their randomized assignment was immense disappointment, followed by a sense of competition and even resentment against the faceless facilitated practices. They then described feelings of resignation, acceptance, and finally, relief and pride that they were able to figure out the journey on their own terms. As one physician in this group put it simply, "I'm happy I got to do things my way."

\section{Self-Organized Retreat (June 2007)}

Many self-directed practices identified a self-organized retreat held midway through the project as a turning point in their journey. Six months into the NDP, one highly motivated self-directed physician contacted all other practices by e-mail, inquiring about interest in a retreat that would allow them to meet, connect, and support each other. The response was overwhelmingly positive, and the practices worked together through e-mail to share responsibility. They asked for and received partial funding from TransforMED; they secured a retreat destination and handled all related logistics; and they built their own agenda of presentations and discussions, serving as the consultants and guest speakers themselves.

The 2-day retreat was held in June 2007. A lead physician and practice manager attended from most practices. No representatives from TransforMED attended, but a member of the evaluation team (E.E.S.) documented the meeting and presented details of the evaluation methods to the self-directed practices, which until that time had almost no knowledge of how the project was being evaluated. The retreat included personal stories to introduce each practice in addition to participant-led presentations and discussions on topics such as same-day scheduling, HIT implementation, clinical care teams, group visits, and wellness promotion. The agenda also included a group discussion on the FFM report and how the guidelines and recommendations translated into the reality of the self-directed practices.

\section{Second Year}

After the retreat, the self-directed practices communicated only sporadically by e-mail. Some practices with similar interests or goals developed deeper communications outside the group format, and 2 physicians collaborated on a paper about the self-directed experience. ${ }^{15}$ Despite the light contact, almost all of these practices attended the final learning session in Kansas City (described below) and publicly affirmed the power of the connections established in that midway retreat. As one self-directed physician stated, "Some of these people, I've met only once ... and yet I feel like I've known them my whole life!"

During the second year, the self-directed practices had more contact with the evaluation team as one member (E.E.S.) visited each practice for a 2- to 3day site visit. Although the purpose of the visit was data collection, not change facilitation, many practice leaders indicated the visit was motivating because it reminded the entire practice they were part of a highprofile project.

\section{Final Learning Session (April 2008)}

The 2 groups of practices finally met face to face and shared experiences during the last NDP learning ses- 
sion. The facilitated practices described feelings of intense pressure, a by product of facilitation and the national spotlight, but also recalled a sense of "feeling pampered" by TransforMED and expressed concern about their future as "self-directed practices." The original self-directed practices remembered their feelings of disappointment and isolation, followed by the realization and pride that they were representing all practices trying to make transformative changes on their own. The facilitated practices appeared to be particularly intrigued by the resourcefulness and strength of the self-directed practices, and the self-directed practices enjoyed sharing some of the "outside-thebox" approaches they took with the FFM model. For example, one night after dinner, a self-directed physician began answering questions about his unusual style of group visits and within minutes, he was giving a hands-on demonstration with mock patients. On the final day, the practices enthusiastically endorsed the notion of staying loosely connected as some kind of "NDP veterans" group.

\section{Epilogue: The Touchstone Group}

The notion of an NDP veterans group quickly emerged as the Touchstone Group, and there was equal interest from both the facilitated and self-directed practices-mostly physicians but also some practice managers. TransforMED provided support in the form of a listserv, open telephone lines for conference calls, limited assistance for an annual reunion meeting, and a year-long free membership to TransforMED's Delta Exchange, an online social networking program. Several Touchstone Group physicians committed to reaching a broader audience by participating in public speaking and writing about their experiences. Many practices collaborated on a letter to NCQA expressing concerns about the medical home recognition process, which eventually led to a face-to-face meeting with NCQA leadership and the opportunity to provide input on future revisions.

The majority of Touchstone Group practices expressed a desire to keep meeting on a yearly basis as a way to reconnect, reflect, and learn from each other. About one-third of the practices reunited in a self-organized retreat in October 2009 for 3 days of sharing against the backdrop of rural Maine's beauty. A representative of TransforMED and a member of the evaluation team (W.L.M.) also attended. It was apparent that the Touchstone attendees had emerged from a profound, shared, life-changing experience, and were unwilling to return to pre-NDP ways of practicing medicine. They had continued developing their practices within what they perceived as a hostile environment and now met for renewal, support, and inspiration.
An overarching theme of the retreat concerned how to get outside the constraints of the current health care system and off of the encounter-based productivity wheel and into a practice home focused on meaningful quality and appropriate care. Many of the practices presented highlights of their past year's work and challenges. Three areas in particular stimulated healthy tension and dialogue-the meaning of teams, connecting to local community, and balancing quality and personality. Attendees discussed what really is a primary care team and who is on it? How do you provide both team care and personal care? How do you increase connection to community agencies, businesses, and other institutions in ways that promote health? How do we ensure excellence in care that is based on the best available evidence and delivered in ways that inspire confidence and satisfaction? As everyone returned home, hopeful for another future reunion, the questions hovered, unanswered inspirational touchstones toward next steps.

\section{DISCUSSION}

The goal of the NDP was to provide proof of concept of the FFM recommendations by using real-life practices to generate transferable new knowledge about practice transformation. To generate this knowledge, the NDP was designed not as a protocol-constrained randomized controlled trial, but as a demonstration project that attempted to do everything reasonably possible to implement the NDP model in facilitated practices. Consequently, both the target model and the intervention strategy evolved as all the key stakeholders learned. ${ }^{16}$ For example, the experience of the facilitated practices seemed to influence the model progression; their requests, questions, and challenges helped to shape the facilitation strategies. The majority of the NDP practices, both facilitated and selfdirected, were exceptionally motivated, especially their practice leaders, resulting in continual learning and insights into the transformation process. ${ }^{17}$ These practices coevolved with their facilitators, illustrating the interdependencies and dynamic nature of change within the complex adaptive system that is a primary care practice of the 21 st century. ${ }^{18}$

The NDP represents the first large-scale implementation of the PCMH model components in diverse primary care practices. The intentional fluidity of the intervention allowed for the natural emergence of knowledge and adaptation to changing events. Many of the lessons are readily transferable to the thousands of practices trying to make changes on their own. At the same time, the complex, evolving intervention, based on realtime evaluation and highly motivated participating prac- 
tices (and their leadership), represents a highly selected situation, and the pace and magnitude of change may not be readily replicated in a more general population.

None of the NDP practices - whether facilitated or self-directed-saw a change in their payment structure. The NDP is noteworthy for providing a look at what highly motivated practices can do to implement $\mathrm{PCMH}$ model components without a direct incentive of payment reform. This lack of financial incentives is also a limitation, as there is no way to know how these practices might have approached change or which changes would have received their greatest attention if they were linked to enhanced reimbursement. Further evaluation of the many PCMH demonstration projects currently in the field will hopefully fill in some of this information. An additional limitation of the NDP for other PCMH demonstration projects is the intense full-court press provided by TransforMED and its facilitators. The NDP also had an embedded evaluation team composed of senior investigators with considerable expertise in qualitative methods and organizational change. It is not clear how much of this infrastructure limits generalizability; however, having a real-time process evaluation, including an embedded team member in the home office, appears to be desirable for providing direct and intensive observation of the intervention as it unfolds.

The NDP model has continued to evolve even after our formal evaluation period ended, and the configuration of components and relative emphasis on particular aspects of the model will likely change accordingly. We expect that current and future research and demonstration projects will learn from TransforMED's evolving model, building on the expanding base of knowledge and boldly moving forward in the spirit of the original NDP practices.

To read or post commentaries in response to this article, see it online at http://www.annfammed.org/cgi/content/full/8I suppl_1/s21.

Key words: Primary health care; family practice; change, organizational; quality of health care; patient-centered care; National Demonstration Project; patient-centered medical home; practice based research

Submitted October 19, 2009; submitted, revised, February 9, 2010; accepted February 23, 2010.

Author affiliations: National Research Network, American Academy of Family Physicians, Leawood, Kansas (at the time of the project, Department of Family and Community Medicine, University of Texas Health Science Center at San Antonio) (Stewart); Center for Research Strategies, Denver, Colorado, and Department of Family Medicine, University of Colorado Health Sciences Center, Aurora, Colorado (Nutting); Department of Family Medicine, Robert Wood Johnson Medical School, University of Medicine $\&$ Dentistry of New Jersey, New Brunswick; Department of Epidemiology, School of Public Health, University of
Medicine $\&$ Dentistry of New Jersey, Piscataway; and Cancer Institute of New Jersey, New Brunswick, New Jersey (Crabtree); Departments of Family Medicine, Epidemiology \& Biostatistics, and Sociology, and The Case Comprehensive Cancer Center, Case Western Reserve University, Cleveland, Ohio (Stange); Department of Family Medicine, Lehigh Valley Health Network, Allentown; and Department of Family and Community Medicine, Pennsylvania State College of Medicine, Hershey, Pennsylvania (Miller); Departments of Family $\&$ Community Medicine, and of Epidemiology \& Biostatistics, University of Texas Health Science Center at San Antonio, San Antonio, Texas (Jaén).

Disclaimer: Drs Stange and Nutting, who are editors of the Annals, were not involved in the editorial evaluation of or decision to publish this article.

Funding support: The independent evaluation of the National Demonstration Project (NDP) practices was supported by the American Academy of Family Physicians (AAFP) and The Commonwealth Fund. The Commonwealth Fund is a national, private foundation based in New York City that supports independent research on health care issues and makes grants to improve health care practice and policy.

Publication of the journal supplement is supported by the American Academy of Family Physicians Foundation, the Society of Teachers of Family Medicine Foundation, the American Board of Family Medicine Foundation, and The Commonwealth Fund.

Dr Stange's time was supported in part by a Clinical Research Professorship from the American Cancer Society.

Disclaimer: The views presented here are those of the authors and not necessarily those of The Commonwealth Fund, its directors, officers, or staff.

Acknowledgments: The National Demonstration Project was designed and implemented by TransforMED, LLC, a wholly-owned subsidiary of the AAFP. We are indebted to the participants in the National Demonstration Project and to TransforMED for their tireless work.

\section{References}

1. Martin JC, Avant RF, Bowman MA, et al; Future of Family Medicine Project Leadership Committee. The future of family medicine: a collaborative project of the family medicine community. Ann Fam Med. 2004;2(Suppl 1):S3-S32.

2. Spann SJ; Task Force 6 and the Executive Editorial Team. Report on financing the new model of family medicine. Ann Fam Med. 2004;2(Suppl 3):S1-S21.

3. Green LA, Graham R, Bagley B, Kilo CM, Spann SJ, Bogdewic SP. Task Force 1. Report of the task force on patient experience, core values, reintegration, and the new model of family medicine. Ann Fam Med. 2004;2(Suppl 1):S33-S50.

4. Jaén $C R$, Ferrer RL, Miller WL, et al. Patient outcomes at 26 months in the patient-centered medical home National Demonstration Project. Ann Fam Med. 2010;8(Suppl 1):s57-s67.

5. Nutting PA, Crabtree BF, Miller WL, Stewart EE, Stange KC, Jaén CR. Journey to the patient-centered medical home: a qualitative analysis of the experiences of practices in the National Demonstration Project. Ann Fam Med. 2010;8(Suppl 1):s45-s56.

6. Nutting PA, Crabtree BF, Stewart EE, et al. Effect of facilitation on practice outcomes in the National Demonstration Project model of the patient-centered medical home. Ann Fam Med. 2010;8(Suppl 1): s33-s44. 
7. Davidoff F, Batalden P, Stevens D, Ogrinc G, Mooney SE. Publication guidelines for quality improvement studies in health care: evolution of the SQUIRE project. BMJ. 2009;338:a3152.

8. Jaén $C R$, Crabtree BF, Palmer $R$, et al. Methods for evaluating practice change toward a patient-centered medical home. Ann Fam Med. 2010;8(Suppl 1):s9-s20.

9. Fetterman D, Wandersman A. Empowerment evaluation: yesterday, today, and tomorrow. Am J Eval. 2007;28(2):179-198.

10. American Academy of Family Physicians (AAFP), American Academy of Pediatrics (AAP), American College of Physicians (ACP), American Osteopathic Association (AOA). Joint principles of the patient-centered medical home. February 2007. http://www.aafp.org/pcmh/ principles.pdf. Accessed Oct 7, 2009.

11. Davis K, Schoenbaum SC, Audet AM. A 2020 vision of patient-centered primary care. J Gen Intern Med. 2005;20(10):953-957.

12. Reid RJ, Fishman PA, Yu O, et al. Patient-centered medical home demonstration: a prospective, quasi-experimental, before and after evaluation. Am J Manag Care. 2009;15(9):e71-e87.
13. Shih A, Davis K, Schoenbaum SC, Gauthier R, Nuzum R, McCarthy D. Organizing the U.S. Health Care Delivery System For High Performance. New York, NY: The Commonwealth Fund; 2008.

14. Stewart EE, Johnson BC. Improve office efficiency in mere minutes. Fam Pract Manag. 2007;14(6):27-29.

15. Loxterkamp D, Kazal LA Jr. Changing horses midstream: the promise and prudence of practice redesign. Ann Fam Med. 2008;6(2): 167-170.

16. Cohen DJ, Crabtree BF, Etz RS, et al. Fidelity versus flexibility: translating evidence-based research into practice. Am J Prev Med. 2008:35(5 Suppl):S381-S389.

17. Nutting PA, Miller WL, Crabtree BF, Jaen CR, Stewart EE, Stange KC. Initial lessons from the first National Demonstration Project on practice transformation to a patient-centered medical home. Ann Fam Med. 2009;7(3):254-260.

18. Miller WL, Crabtree BF, Nutting PA, Stange KC, Jaén CR. Primary care practice development: a relationship-centered approach. Ann Fam Med. 2010;8(Suppl 1):s68-s79. 